\title{
Expresión de Citoqueratina 5 y Calretinina en el carcinoma renal de células claras
}

\author{
Cid Mouteira P, Ortiz-Rey JA, Gómez de María C, San Miguel Fraile P, Albor Barroso A, \\ Regueiro Parcero B, Antón Badiola I.
}

Servicio de Anatomía Patológica. Hospital Povisa. Vigo (Pontevedra).

Actas Urol Esp 2006; 30 (4): 367-371

\section{RESUMEN}

EXPRESIÓN DE CITOQUERATINA 5 Y CALRETININA EN EL CARCINOMA RENAL DE CÉLULAS CLARAS

Objetivo: La citoqueratina 5 (CK5) y la calretinina se han revelado en numerosos estudios como marcadores inmunohistoquímicos sugestivos de mesotelioma y su análisis se utiliza para el diagnóstico diferencial histológico con adenocarcinomas, especialmente ante metástasis de origen desconocido. En el presente estudio hemos analizado la expresión de CK5 y calretinina en el carcinoma renal de células claras.

Métodos: Se estudió un total de 63 casos de carcinoma renal de células claras. De ellos, 46 fueron incluidos en dos matrices tisulares ("tissue arrays"), y un segundo grupo, de 17 casos, estuvo constituido por bloques convencionales de tumores de alto grado (grado 4 de Fuhrman).

Se realizaron tinciones inmunohistoquímicas con anticuerpos monoclonales anti CK5 y anti calretinina, mediante el método de estreptavidina marcada con peroxidasa-biotina.

Resultados: No se obtuvo positividad en ninguno de los casos para calretinina, mientras que la CK5 se expresó focalmente, en células aisladas, en 1 de los 63 casos (1,59\%), que correspondió a un carcinoma de alto grado (grado 4 de Fuhrman).

Conclusiones: En el carcinoma renal de células claras no hemos observado expresión de calretinina y la positividad para CK5 ha ocurrido de manera aislada y en un porcentaje muy pequeño de células tumorales. Por tanto, en la práctica si bien la positividad para estos marcadores no excluye de manera absoluta carcinoma de células renales, dicho resultado es muy improbable en este tumor y obliga a considerar otras opciones diagnósticas.

Palabras Clave: Carcinoma renal. Carcinoma de células claras. Citoqueratina 5. Calretinina. Inmunohistoquimica.

\section{ABSTRACT}

EXPRESSION OF CYTOKERATIN 5 AND CALRETININ IN CLEAR CELL RENAL CELL CARCINOMA

Purpose: Cytokeratin 5 (CK5) and calretinin have been useful in different studies as immunohistochemical markers suggestive of mesothelioma, and their expression is analyzed for the histological differential diagnosis with adenocarcinomas, specially when confronting with metastatic tumors of unknown origin. We have analyzed the expression of CK5 and calretinin in clear cell renal cell carcinoma.

Methods: A series of 63 clear cell renal cell carcinomas was studied. 46 of these cases were embedded in two tissue arrays, and a second group, of 17 cases, was constituted by conventional paraffin blocks from high-grade tumors (grade 4 of Fuhrman).

Immunohistochemical staining was performed with monoclonal antibodies against CK5 and calretinin, following the labeled sptreptavidin-biotin technique.

Results: No positivity for calretinin was observed in any case, while CK5 was focally expressed, in an isolated group of cells, in 1 of the 63 cases (1,59\%) which corresponded to a high-grade carcinoma (grade 4 of Fuhrman).

Conclusions: Expression of calretinin was not observed in clear cell renal cell carcinoma and positivity for CK5 occurred only in one case, in a very small proportion of tumor cells. Therefore, in practice, although the positivity for these markers cannot completely exclude renal cell carcinoma, this result is very rare in this tumor and other diagnostic posibilities should be considered. 
$\mathrm{E}$ 1 carcinoma renal de células claras se caracteriza por producir, en ocasiones, metástasis en localizaciones inusuales (piel, bazo, corazón, tubo digestivo, etc), que pueden ser, además, de presentación tardía ${ }^{1}$. Por ello, en ocasiones se plantea el diagnóstico diferencial histológico con otras neoplasias, entre las que se puede incluir el mesotelioma, especialmente en su variante de células claras ${ }^{2,3}$

La CK5 (Citoqueratina 5) y la calretinina están considerados por numerosos autores como valiosos marcadores inmunohistoquímicos de mesotelioma $^{2,4}$.

La CK5 forma parte de una familia de unos 20 polipéptidos constituyentes del citoesqueleto celular, cuya clasificación numérica fue propuesta por Moll et al., basándose en su peso molecular y punto isoeléctrico ${ }^{5}$. En los epitelios normales la expresión de CK5 se encuentra restringida a las células basales de los epitelios glandular y escamoso, mioepitelio, epitelio escamoso y mesotelio $^{5}$. Debido a esta especificidad y a la particularidad de que las células epiteliales mantienen su patrón de citoqueratinas durante la malignización y metástasis $^{6}$, es posible la tipificación inmunohistoquímica de mesoteliomas mediante la determinación de CK5.

La calretinina es una proteína ligadora del calcio que tiene función tampón al prevenir un aumento excesivo de calcio intracelular ${ }^{7}$. Se expresa abundantemente en el sistema nervioso central y periférico ${ }^{7,8} \mathrm{y}$, en menor medida, en otras localizaciones como ovario ${ }^{9}$. Diversos estudios han demostrado una altísima especificidad de la calretinina para las células del mesotelio tanto normal como maligno ${ }^{10}$.

En la literatura son escasos los trabajos que han analizado la expresión de calretinina y CK5 en el carcinoma renal y algunos de ellos con un número muy limitado de casos. La mayoría de los estudios ponen en evidencia una baja frecuencia en la expresión de ambos marcadores aunque existen discrepancias en los resultados $2,4,12,13$

Nuestro objetivo es analizar la expresión de CK5 y calretinina en un grupo de carcinomas renales en su variante de células claras.

\section{MATERIAL Y MÉTODOS}

El presente estudio incluye un total de 63 casos de carcinoma renal de células claras, (10 de grado 1, 24 de grado 2,8 de grado 3 y 21 de grado 4 , según la clasificación de Fuhrman ${ }^{11}$ diagnosticados en el Servicio de Anatomía Patológica del Hospital POVISA.

Un primer grupo de casos correspondió a 46 bloques de parafina a partir de los cuales se elaboraron dos matrices tisulares ("tissue arrays") extrayéndose dos cilindros de zonas representativas seleccionadas de cada uno de los bloques "donantes" con aguja de calibre $16 \mathrm{G}$.

En una segunda fase, debido a los resultados preliminares obtenidos en el primer grupo, el estudio se amplió con bloques de parafina convencionales de 17 carcinomas renales de alto grado (grado 4 de Fuhrman).

Todos los casos habían sido fijados en formol neutro al $10 \%$, sometidos a un procesado rutinario e incluidos en parafina. Se estudiaron secciones de 4 micras de cada bloque, tanto de los convencionales como de las matrices tisulares. El desenmascaramiento antigénico se realizó en olla a presión durante 5 minutos, en tampón citrato $0.01 \mathrm{M}$ a pH 6. Posteriormente se llevó a cabo una inhibición de la peroxidasa endógena en peróxido de hidrógeno al 3\% durante 10 minutos. Se incubaron los anticuerpos primarios anti CK5 $\mathrm{y}$ anti calretinina durante 30 minutos a temperatura ambiente. Los datos de los anticuerpos utilizados se resumen en la Tabla 1. El método de detección fue el de la estreptavidina marcada con peroxidasa - biotina (LSAB2®, DakoCytomation, Dinamarca). El revelado se realizó con diaminobencidina (DAB), con lo que la positividad consistió en tinción de color marrón.

Tabla 1

Especificaciones de los anticuerpos utilizados

\begin{tabular}{lcc}
\hline Anticuerpo & Dilución & Clon, Fabricante \\
CK5 & $1: 100$ & XM26, Novocastra \\
Calretinina & $1: 40$ & 5 A5, Novocastra \\
\hline
\end{tabular}

Se incluyeron controles positivos y negativos para asegurar la fiabilidad de la técnica.

\section{RESULTADOS}

En las dos matrices tisulares preparadas (46 casos) se obtuvo negatividad en la expresión de CK5 y Calretinina (Fig. 1). 


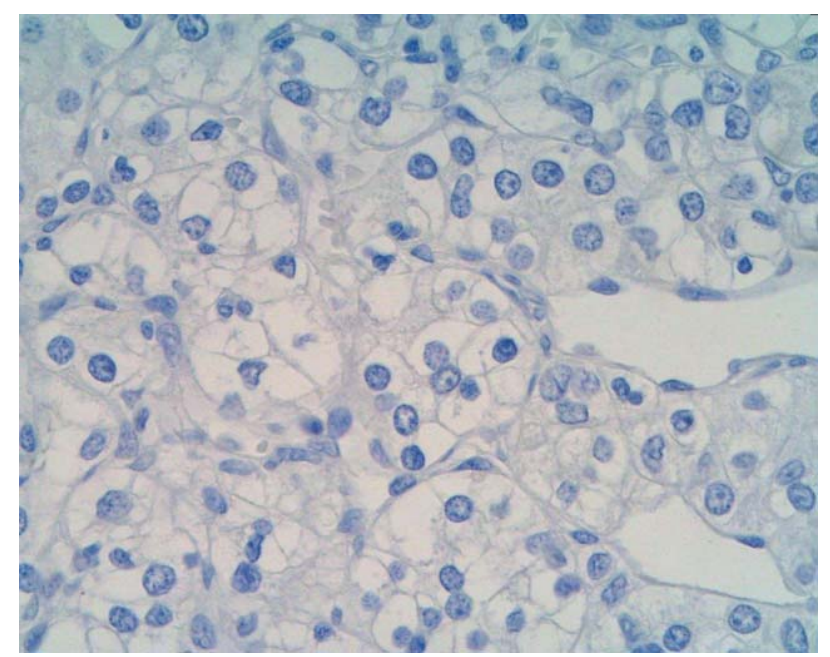

FIGURA 1: Tinción para calretinina negativa en carcinoma renal de células claras. (Inmunoperoxidasa, x400).

En el segundo grupo de 17 carcinomas de alto grado (grado 4 de Fuhrman), únicamente hubo expresión de CK5 en un caso (Fig. 2). Cabe destacar que dicha positividad fue muy focal, en menos del $1 \%$ de las células.

Por lo tanto, ninguno de los casos de ambos grupos fue positivo para calretinina, y la expresión de CK5 se encontró en un 1,59\% (1/63) de los casos estudiados, y en células aisladas.

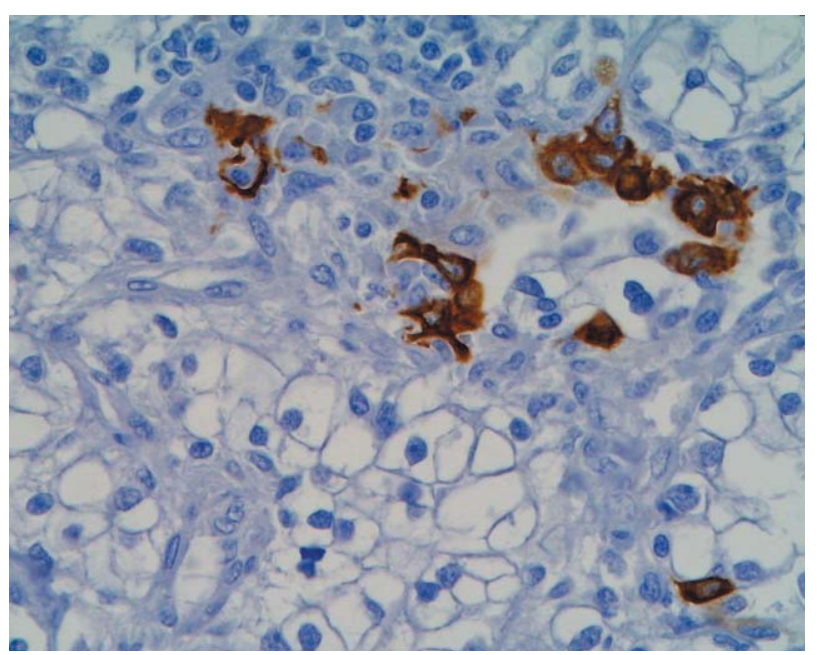

FIGURA 2: Positividad para CK5 en escasas células en un carcinoma renal de células claras. (Inmunoperoxidasa, x400).

con menos de 10 casos estudiados ${ }^{10,12-14}$, y en la de Ordoñez ${ }^{4}$ con 48 casos, apareciendo, sin embargo, positividad en otras series ${ }^{2,15,16}$ con un porcentaje de expresión que varía entre un 4 y un $10 \%$ (entre un 1,3 y un $10 \%$ si nos ceñimos a la variante de células claras).

Osborn et al. analizan 40 casos de carcinoma renal, de los cuales 30 son sólidos, o mixtos con áreas fusocelulares, que considera como carcino-

\section{DISCUSIÓN}

La CK5 y la calretinina son marcadores sensibles de mesotelioma y se han convertido en componentes fundamentales de los paneles de inmunohistoquímica normalmente utilizados para el diagnóstico diferencial entre mesotelioma y adenocarcinoma $^{2,4,10,12}$.

Ambos aparecen como claros marcadores positivos de mesotelioma en oposición a diversos tipos tumorales, entre los que se incluye el carcinoma renal en algunos estudios que comparan ambas neoplasias ${ }^{2,4}$.

La expresión de calretinina en el carcinoma renal se ha analizado en varios trabajos, que se resumen en la Tabla 2. Se observa que la calretinina ha sido constantemente negativa en todas las series

Tabla 2

Expresión de Calretinina y CK5 en carcinoma renal en diferentes estudios

\begin{tabular}{lcc}
\hline Estudio & Calretinina & CK5 \\
\hline Ordóñez $^{4}$ & $0 / 48$ & $0 / 48$ \\
Osborn et al. $^{2}$ & $4 / 40$ & $2 / 40$
\end{tabular}

(3/30 carcinomas renales de células claras)

Doglioni et al. ${ }^{10}$

Attanoos et al. ${ }^{12}$

Cury et al. ${ }^{13}$

Ordóñez ${ }^{14}$

Lugli et al. ${ }^{15}$

Martignoni et al. ${ }^{16}$

Ordóñez ${ }^{17}$

Chu et al. ${ }^{18}$

Presente estudio

\section{$0 / 4$ \\ $0 / 1$ \\ $0 / 4$ \\ $0 / 8$}

$7 / 143$

(3/89 carcinomas renales de células claras)

$5 / 124$

(1/75 carcinomas renales de células claras)
(0/30 carcinomas renales de células claras)
$0 / 1$
$1 / 4$

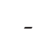

$\begin{array}{ll}- & 0 / 10 \\ - & 0 / 19 \\ 0 / 63 & 1 / 63\end{array}$


mas de células claras ${ }^{2}$. De los 30 casos, 6 presentan positividad a calretinina, pero únicamente considera valorables los casos con tinción nuclear, que sería más específica que la citoplasmática. Resultan así 3 casos positivos de los 30 estudiados, correspondientes a grados 2 y 3 , y con un porcentaje de células reactivas que varia entre 1 y $50 \%$ de las células tumorales.

Martignoni et al. estudian 124 casos de carcinoma renal de distintos grados nucleares, de los cuales 75 corresponden a la variante de células claras. Sólo se obtiene en un caso expresión de calretinina débil y focal ${ }^{16}$.

Lugli et al. analizan 143 casos de carcinoma renal, especificando que 89 de ellos son carcinomas de células claras, de los cuales 3 expresan calretinina ( 2 con positividad débil y 1 con intensa positividad) ${ }^{15}$.

En el presente estudio hemos analizado una serie de 63 casos de carcinoma renal de células claras de distintos grados nucleares. Al haber sido negativos los 46 casos que constituían las matrices tisulares ("tissue arrays"), seleccionamos un segundo grupo de tumores de alto grado (17 casos), en los que cabría esperar una mayor probabilidad de expresión aberrante de marcadores tisulares, debido al menor grado de diferenciación. Tampoco se encontró expresión de calretinina en ningún caso.

Nuestros resultados son por tanto comparables con algunas de las series expuestas. La expresión de calretinina se muestra en todas ellas por debajo del 10\% de los casos estudiados, reduciéndose este porcentaje si nos centramos en los casos de carcinoma renal de células claras.

La CK5 presenta una expresión limitada a epitelios glandular, escamoso, mioepitelio y mesote$\mathrm{lio}^{5}$, lo que se traduce en un alto grado de especificidad tisular y convierte a este anticuerpo en un excelente marcador mesotelial ${ }^{2,4,8}$.

En los estudios que han analizado la expresión de CK5 en los carcinomas renales aquella se observa con muy baja frecuencia (Tabla 2) 2,4,12,13,17,18. Únicamente Cury et al. presentan un caso positivo de 4 carcinomas renales, aunque sin especificar la histología del tumor ${ }^{13}$.

Osborn et al. han encontrado 2 casos positivos de 40 carcinomas renales, ninguno de ellos de la variante de células claras ${ }^{2}$.
En nuestro estudio, se obtuvo un único caso de positividad entre los 63 estudiados. Hay que destacar que la tinción ha sido focal y aislada, prácticamente restringida a un pequeño grupo celular, y en un tumor de alto grado (grado 4). Estos resultados y los de otras series en las que la expresión de CK5 se da mayoritariamente en carcinomas de grado $3^{2}$, hace pensar que es en aquellos tumores poco diferenciados en los que puede aparecer con mayor probabilidad expresión de CK5.

De nuestros resultados y de la revisión de la literatura se concluye que la expresión de calretinina y CK5 resulta muy infrecuente en el carcinoma renal de células claras, por lo que la positividad para ambos marcadores obliga a considerar como más probables otras opciones diagnósticas.

\section{REFERENCIAS}

1. Eble JN, Sauter G, Epstein JI, Sesterhenn IA: World Health Organization Classification of Tumours. Pathology and genetics of tumours of the urinary system and male genital organs. International Agency for Research on Cancer Press 2004:23-25.

2. Osborn M, Pelling N, Walker MM, Fisher C, Nicholson AG. The value of "mesothelium-associated" antibodies in distinguishing between metastatic renal cell carcinomas and mesotheliomas. Histopathology 2002;41(4):301-307.

3. Ordóñez NG. Mesothelioma with clear cell features: an ultrastructural and immunohistochemical study of 20 cases. Hum Pathol 2005;36(5):465-473.

4. Ordóñez NG. The diagnostic utility of immunohistochemistry in distinguishing between mesothelioma and renal cell carcinoma: a comparative study. Hum Pathol 2004; 35(6):697-710.

5. Moll R, Franke WW, Schiller DL, Geiger B, Krepler R. The catalog of human cytokeratins: patterns of expression in normal epithelia, tumors and cultured cells. Cell. 1982; 31(1):11-24.

6. Hynes RO, Destree AT. $10 \mathrm{~nm}$ filaments in normal and transformed cells. Cell. 1978;13(1):151-163.

7. Rogers JH. Calretinin: A gene for a novel calcium-binding protein expressed principally in neurons. J Cell Biol 1987; 105: 1343-1353.

8. Gown AM, Bacchi CE. Diagnostic immunohistochemistry of solid tumors: strategies and solutions; 2002 syllabus short course \#35. United States and Canadian Academy of Pathology 2002:43-49.

9. Bertschy S, Genton CY, Gotzos V. Selective immunocytochemical localisation of calretinin in the human ovary.Histochem Cell Biol. 1998;109(1):59-66.

10. Doglioni C, Tos AP, Laurino L, Iuzzolino P, Chiarelli C, Celio MR, Viale G. Calretinin: a novel immunocytochemical marker for mesothelioma. Am J Surg Pathol. 1996 20(9): 1037-1046.

11. Fuhrman SA, Lasky LC, Limas C. Prognostic significance of morphologic parameters in renal cell carcinoma. Am $\mathrm{J}$ Surg Pathol 1982;6(7):655-663. 
12. Attanoos RL, Dojcinov SD, Webb R, Gibbs AR. Anti-mesothelial markers in sarcomatoid mesothelioma and other spindle cell neoplasms. Histopathology. 2000;37(3):224231

13. Cury PM, Butcher DN, Fisher C, Corrin B, Nicholson AG. Value of the mesothelium-associated antibodies thrombomodulin, cytokeratin 5/6, calretinin, and $\mathrm{CD} 44 \mathrm{H}$ in distinguishing epithelioid pleural mesothelioma from adenocarcinoma metastatic to the pleura. Mod Pathol 2000; 13(2):107-112.

14. Ordóñez NG. Value of calretinin immunostaining in differentiating epithelial mesothelioma from lung adenocarcinoma. Mod Pathol. 1998;11(10):929-33.

15. Lugli A, Forster Y, Haas P, Nocito A, Bucher C, Bissig H, et al. Calretinin expression in human normal and neoplastic tissues: a tissue microarray analysis on 5233 tissue samples. Hum Pathol. 2003;34(10):994-1000.
16. Martignoni G, Pea M, Chilosi M, Brunelli M, Scarpa A, Colato $\mathrm{C}$, et al. Parvalbumin is constantly expressed in chromophobe renal carcinoma. Mod Pathol 2001;14(8):760-767.

17. Ordóñez NG. Value of cytokeratin 5/6 immunostaining in distinguishing epithelial mesothelioma of the pleura from lung adenocarcinoma. Am J Surg Pathol 1998;22(10):12151221.

18. Chu PG, Weiss LM. Expression of cytokeratin 5/6 in epithelial neoplasms: An immunohistochemical study of 509 cases. Mod Pathol. 2002 Jan;15(1):6-10.

Dr. J.A. Ortiz Rey.

Anatomía Patológica. POVISA.

Salamanca, 5 - 36211 Vigo (Pontevedra)

e-mail: jaortiz@povisa.es

(Trabajo recibido el 19 de diciembre 2005) 